\title{
Über Calciumsilicide und deren Aufnahmefähigkeit für Stickstoff.
}

\author{
Von \\ A. Kolb.
}

Das Verhalten des Calciums gegen Stickstoff hat mich veranlalst, einige Verbindungen dieses Metalles in gleicher Richtung zu untersuchen. Die Versuche wurden mit dem Calciumsilicid, von dem jedenfalls die Fähigkeit zu erwarten war, Stickstoff bei höherer Temperatur aufzunehmen, begonnen, weil die andere Komponente für sich allein auch hierzu imstande ist. Herr Richand Formuals hat auf meine Veranlassung diese Arbeit unternommen. Im nachstehenden soll ein Auszug aus seiner Dissertation mitgeteilt werden. Die bisher bekannten Darstellungsmethoden ${ }^{1}$ für Calcium. silicid liefern ein unreines und für unsere Zwecke ungeeignetes Material. Wir gingen deshalb von metallischem Calcium und reinem Silicium aus, von denen uns ersteres von den Elektrochemischen Werken Bitterfeld in liebenswürdiger Weise zur Verfügung gestellt wurde. Die Zusammensetzung des Silicids wurde von den genannten Autoren gewöhnlich durch die Formel $\mathrm{CaSi}_{2}$ ausgedrückt. Es überraschte uns daher etwas, als wir bei unserem Silicid nach der Analyse zu einer anderen Formel gelangten. Die Eigenschaften desselben waren indessen keine wesentlich anderen. Es mulste deshalb die Untersuchung in drei Abschnitte zergliedert werden, von denen sich der erste mit der Zusammensetzung und den Eigenschaften des Silicids, der zweite mit den daraus erhältlichen Silikonen und der dritte schliefslich mit der Aufnahmefähigkeit des Silicids für Stickstoff befafste. Nach Beendigung der Untersuchung des Silicids erschien die Mitteilung von S. TAMARU ${ }^{2}$ uber Silicium-Calciumlegierungen.

1 Wö̈ler, Lieb. Ann. 125, 255; 127, 257. - DE Chalyot, Am. Chem. Journ. 18 (1896), 314; Zeitsehr. f. Elektrochem. 3, 83. - Bradoley, Chem. Neucs 82, 149. - Morgsan und Dilthex, Ber. 35, 1106. - Jungst und Mewes, D.R.P. 157615; Centrbl. 1905 I, 194. - Goldschuidr, D.R.P. 199193 , Centrbl. 1908 II, 271 und D.R.P. 204567; Centrbl. 1909 I, 112; Soc. générale delektro-chimie de Bozel, D.R.P. 206785 ; Centrbl. 1909 I, 1126.

${ }^{2} Z$. anorg. Chem. 62, 81-88. 
Der Autor suchte die Frage der Zusammensetzung auf dem Wege der thermischen Analyse zu entscheiden und stellte hierbei fest, dafs flüssiges Calcium und flüssiges Silicium miteinander in allen Verbältnissen mischbar sind, und dafs bis etwa $35 \% \mathrm{Si}$ sich praktisch reines Silicium ausscheidet, das bei $990^{\circ}$ mit der etwa $35 \%$ Si-haltigen Schmelze die Verbindung $\mathrm{CaSi}_{2}$ zu bilden scheint. Weitere Verbindungen von Calcium und Silicium konnte Tamaro nicht konstatieren, stellt aber deren Existenz nicht als ausgeschlossen hin.

\section{Darstellang der Silicide.}

Das verwendete Calcium enthielt $96-97 \%$ reines $\mathrm{Ca}$, etwa $1 \% \mathrm{CaO}, 1.2 \% \mathrm{Fe}$ und $\mathrm{Al}$, etwas $\mathrm{SiO}_{2}$ und manchmal auch etwas Kohle. Das Silicium war rein. Durch Zusammenschmelzen ron Silicium und Calcium konnten zwei Silicide erhalten werden, je nachdem die eine oder die andere Komponente im Überschufs verwendet wurde. Das aus Calcium und überschüssigem Silicium erhaltene Produkt, das in seinen Eigenschaften mit dew WöHLkrschen Silicid übereinstimmte, soll weiterhin mit silicid I, das andere mit überschüssigem Calcium erhaltene Produkt mit Silicid II bezeichnet werden.

Silicid I. Beim einfachen Zusammenschmelzen der beiden Komponenten in dem der Formel $\mathrm{CaSi}_{2}$ entsprechenden Verhältnis bleibt viel Silicium unverbunden, denn der Körper enthält mehr Calcium, als die Formel verlangt. Aulserdem war kein koustantes Produkt auf diese Weise zu erhalten, dagegen liefsen sich auf die folgende Art annähernd gleiche Produkte darstellen. Das Calcium wurde in möglichst dünne Blättchen ausgeschlagen und in einem Porzellantiegel in überschüssiges, fein pulverisiertes Silicum eingebettet. In den Tiegel wurde ein kräftiger Wasserstoffstrom geleitet. Da die Reaktion erst bei ziemlich hoher Temperatur vor sich geht, so mufs zur Einleitung der Reaktion mit dem Gebläse erhitzt werden. Die Vereinigung geht unter Feuererscheinung vor sich, wobei die Masse bis zur Weilsglut kommt. Nach dem Erkalten befindet sich in dem Tiegel eine Schlacke, die sich sehr leicht durch Auslesen, grobes Pulverisieren und Absieben von dem überschüssigen Silicium trennen läfst. Bei Anwendung von aluminiumbaltigem Silicium schmolz das Silicid sehr schön zusammen und erstarrte dann krystallinisch. Die so gewonnenen Silicide entbielten $45-49 \% \mathrm{Ca}$, sie wurden einzeln analysiert und die gleichen 
Produkte miteinander gemischt. Die Analyse erfolgte in der Art, dals die pulverisierte Substanz im Tiegel an der Luft verglubt und dann mit reinem Natriumhydroxyd geschmolzen wurde. In der Schmelze ist sodann auf die übliche Weise Si und Ca bestimmt worden. Auf diese Weise wurden folgende Werte gefunden:

1. $0.2303 \mathrm{~g}$ angew. Substanz ergaben $0.2556 \mathrm{~g} \mathrm{SiO}_{2}=\mathbf{5 2 . 1 8} \% \mathrm{Si}$ $0.1487 \mathrm{~g} \mathrm{CaO}=46.16 \% \mathrm{Ca}$ $0.0030 \mathrm{~g} \mathrm{Fe}_{2} \mathrm{O}_{3}=0.91 \% \mathrm{Fe}$

$0.105 \overline{3} \mathrm{~g}$ Substanz entwickelteu beim Kochen mit Salzsäure und darauffolgend mit Natronlauge insgesamt $106.8 \mathrm{ccm}$ Wasserstoff von $0^{0}$ und $760 \mathrm{~mm}, 1 \mathrm{~g}$ demnach $1.014 \mathrm{l}$.

2. $0.1214 \mathrm{~g}$ angew. Substanz ergaben $0.1270 \mathrm{~g} \mathrm{SiO}_{2}=49.19 \% \mathrm{Si}$

$0.0808 \mathrm{~g} \mathrm{CaO}=\mathbf{4 7 . 5 8} \% \mathbf{C a}$

$0.0019 \mathrm{~g} \mathrm{Fe}_{2} \mathrm{O}_{3}=1.09 \% \mathrm{Fe}$

3. $0.1223 \mathrm{~g}$ angew. Substanz ergaben $0.1258 \mathrm{~g} \mathrm{SiO}_{2}=48.37 \% \mathrm{Si}$

$0.0820 \mathrm{~g} \mathrm{CaO}=47.93 \% \mathrm{Ca}$

$0.0017 \mathrm{~g} \mathrm{Fe}_{2} \mathrm{O}_{3}=\mathbf{0 . 9 7 \%} \mathbf{F e}$

4. $0.1329 \mathrm{~g}$ angew. Substanz ergaben $0.1395 \mathrm{~g} \mathrm{SiO}_{2}=49.36 \% \mathrm{Si}$ $0.0880 \mathrm{~g} \mathrm{CaO}=47.34 \% \mathrm{Ca}$

5. $0.1653 \mathrm{~g}$ angew. Substanz ergaben $0.1828 \mathrm{~g} \mathrm{SiO}$ $0.1055 \mathrm{~g} \mathrm{CaO}=45.63 \% \mathrm{Ca}$

6. $0.1048 \mathrm{~g}$ angew. Substanz ergaben $0.1156 \mathrm{~g} \mathrm{SiO}=\mathbf{5 1 . 8 6} \% \mathbf{S i}$ $0.0655 \mathrm{~g} \mathrm{CaO}=44.68 \% \mathrm{Ca}$ $0.0014 \mathrm{~g} \mathrm{Fe}_{2} \mathrm{O}_{3}=0.93 \% \mathrm{Fe}$

$0.1088 \mathrm{~g}$ Substanz entwickelten nacheinander mit Salzsäure und Natronlauge gekocht $112.0 \mathrm{ccm}$ Wasserstoff von $0^{\circ}$ und $760 \mathrm{~mm}$, $1 \mathrm{~g}$ demnach $1.03 \mathrm{l}$.

Für die Beurteilung der Reinheit eines Silicids kommt aufser der Menge der einzelnen Bestandteile noch die Menge Wasserstoff in Betracht, die durch das Silicid bei seiner völligen Zersetzung entwickelt wird. Diese Wasserstoffmenge wurde auf folgende Weise ermittelt:

Fein gepulvertes Silicid wurde in ein kleines trockenes Kölbchen eingewogen und das Kölbchen mit einem doppelt durchbohrten Kork versehen, durch dessen eine Bohrung ein Tropftrichter mit eingeschliffenem Gaszuführungsrobr führte. Durch die andere Bohrung ging ein Gasableitungsrohr, das mit einem für Stickstoff bestimmungen gebräuchlichen Gasabsorptionsapparat in Verbindung stand. In den 
Apparat wurde Kohlensäure geleitet und dadurch die Luft vertrieben. Dann wurde mit Hilfe des Tropftrichters konzentrierte Salzsäure in das Kölbchen gegeben, wobei sich das Silicid unter Bildung von Silikonen und Wasserstoff resp. Siliciumwasserstoff zersetzte. Der Wasserstoff wurde uber konzentrierter Kalilauge aufgefangen, wobei sich auch der Siliciumwasserstoff zersetzte, und zwar nach der Gleichung:

$$
\mathrm{SiH}_{4}+4 \mathrm{KOH}=\mathrm{Si}(\mathrm{OK})_{4}+8 \mathrm{H} .
$$

Man erhält also trotzdem eine dem vorhandenen Silicium äquivalente Wasserstoffmenge. Die Flüssigkeit wurde dann kurz aufgekocht und die Kohlensäure abgestellt. Hierauf wurde Kalilauge in das Kölbchen gegeben, wobei sich die Silikone unter Wasserstoffentwickelung zersetzten. Die Flüssigkeit wurde noch einmal aufgekocht und mit Kohlensäure die letzten Reste Wasserstoff ubergetrieben. Dann wurde der Wasserstoff in eine Eudiometerröhre übergefullt und gemessen. Auf diese Weise wurden die bei den Analysen angegebenen Wasserstoffmengen gefunden.

Nr. 1 ergab, wie angegeben, folgende Werte:

$52.18 \% \mathrm{Si}, 46.16 \% \mathrm{Ca}, 0.91 \% \mathrm{Fe}$ und 1.014 I Wasserstoff pro $1 \mathrm{~g}$. Berechnet man aus den Analysenresultaten die Menge Wasserstoff, die entwickelt werden mürste, so findet man folgende Werte:

$28.4 \mathrm{~g} \mathrm{Si}$ entwickeln nach der Gleichung:

44.81 Gas.

$$
\mathrm{Si}+4 \mathrm{H}_{2} \mathrm{O}=\mathrm{Si}(\mathrm{OH})_{4}+4 \mathrm{H}
$$

$40 \mathrm{~g}$ Ca nach der Gleichung:

22.4 1 Gas.

$$
\mathrm{Ca}+2 \mathrm{H}_{2} \mathrm{O}=\mathrm{Ca}(\mathrm{OH})_{2}+2 \mathrm{H}
$$

$56 \mathrm{~g}$ Fe nach der Gleichung:

22.41 Gas.

$$
\mathrm{Fe}+2 \mathrm{H}_{2} \mathrm{O}=\mathrm{Fe}_{(}\left(\mathrm{OH}_{2}+2 \mathrm{H}\right.
$$

$52.18 \mathrm{~g} \mathrm{Si}$ entwickeln demnach $82.31 \mathrm{l}$

$$
\begin{array}{rlrr}
46.16 \mathrm{~g} \mathrm{Ca} & " & & 25.851 \\
0.91 \mathrm{~g} \mathrm{Fe} & " & & 0.361 \\
\hline 108.521
\end{array}
$$

$100 \mathrm{~g}$ Silicid sollten danach 108.52 I Gas entwickeln, tatsächlich wurden aber nur $101.4 \mathrm{l}$ entwickelt.

Nr. 6 enthielt folgende Mengen:

$51.86 \% \mathrm{Si}, 4468 \% \mathrm{Ca}, 0.93 \% \mathrm{Fe}, 1 \mathrm{~g}$ entwickelte 1.031 Gas. 
Es sollten nach folgenden Ansätzen entwickelt werden:

$$
\begin{array}{lr}
\frac{28.4}{44.8} \mathrm{Si}=\begin{array}{c}
51.86 \\
x
\end{array} & x=81.80 \mathrm{l} \\
\frac{40.0}{22.4} \mathrm{Ca}=\begin{array}{c}
44.68 \\
y
\end{array} & y=25.021 \\
56.0 \mathrm{Fe}=0.93 & z=0.37 \mathrm{l} \\
22.4 & 107.191
\end{array}
$$

$100 \mathrm{~g}$ Silicid sollten demnach $107.19 \mathrm{l}$ Wasserstoff entwickeln, es wurden aber nur $103.0 \mathrm{l}$ entwickelt.

Die Silicide enthalten demnach noch Verunreinigungen, die im wesentlichen nur aus $\mathrm{CaO}$, kohlensaurem und kieselsaurem Kalk bestehen können. Es wurde anfangs versucht, den Kalk durch Auskochen mit vielem Wasser zu entfernen. Doch zeigte es sich, dals dadurch das Silicid stark zersetzt wurde. Die Reinigung gelang dagegen leicht durch Waschen mit eiskaltem Zuckerwasser. Das grob gepulverte Silicid wurde kurze Zeit mit konzentrierter Zuckerlösung stehen gelassen, dann abgesangt, mit kaltem Wasser ausgewaschen und getrocknet. Die Analyse des auf solchem Wege erhaltenen Produktes ergab folgende Werte:

$0.1477 \mathrm{~g}$ angew. Substanz ergaben:

$$
\begin{aligned}
& 0.1660 \mathrm{~g} \mathrm{SiO}_{2}=52.85 \% \mathrm{Si} \\
& 0.0916 \mathrm{~g} \mathrm{CaO}^{2}=44.34 \% \mathrm{Ca} \\
& 0.0020 \mathrm{~g} \mathrm{Fe}_{2} \mathrm{O}_{3}=0.95 \% \mathrm{Fe}
\end{aligned}
$$

$1 \mathrm{~g}$ Substanz entwickelte $1.063 \quad \mathrm{l}$ Wasserstoff ron $\theta^{\prime \prime}$ und $760 \mathrm{~mm}$. Nach den folgenden Ansützen berechnen sich die Werte:

$$
\begin{aligned}
& \begin{array}{l}
28.4 \mathrm{Si} \\
44.8
\end{array}=\frac{52.85}{x} \quad x=83.37 \mathrm{l} \\
& 22.4=\begin{array}{c}
44.34 \\
y
\end{array} \quad y=24.831
\end{aligned}
$$

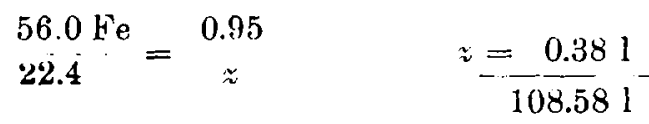

$100 \mathrm{~g}$ Silicid sollten $108.58 \mathrm{lWasserstoff}$ entwickeln. Es wurden aber nur $106.3 \mathrm{l}$ entwickelt.

Nach dem Auswaschen mit Zuckerwasser befinden sich immer noch geringe Verunreinigungen im Silicid, die jetzt nur aus etwas 
kohlensaurem und kieselsaurem Kalk bestehen können, die sich aber, ohne das Silicid zu zersetzen, nicht entfernen lassen. Es müssen also diese Körper bei der Berechnung der Formel mit in Betracht gezogen werden. Aufserdem war noch die Menge an freiem Silicium zu ermitteln. $\mathrm{Zu}$ diesem $\mathrm{Zweck}$ wurde das Silicid mit Flufssäure und Schwefelsäure abgeraucht, der Rückstand mit heifser Salzsäure gewaschen und gewogen.

$0.4194 \mathrm{~g}$ Silicid ergaben dabei einen Rückstand von $0.0092 \mathrm{~g}$ $\mathrm{Si}=2.19 \%$.

Von dem Eisen waren in Salzsäure löslich $0.32 \%$, Eisensilicid von der Formel $\mathrm{FeSi}_{2}$ ist in Salzsäure unlöslich, der Rest des vorbandenen Eisens ist also auf dieses Silicid umzurechnen und die entsprechende Menge Silicium von dem Gesamtsilicium abzuziehen.

Wie aus verschiedenen Umständen hervorgeht, ist die dem Silicid beigemengte Verunreinigung in Salzä̈ure löslich. Sie mufs also im wesentlichen aus einer Calciumverbindung bestehen, und zwar kommen hier nur kohlensaurer und kieselsaurer Kalk in Betracht. Die Berechnung soll für den ersteren Fall durchgeführt werden.

$100 \mathrm{~g}$ Silicid entwickelten $106.3 \mathrm{I}$ Wasserstoff; wie berechnet, sollten sie $108.58 \mathrm{l}$ entwickeln. Fs waren demnach in dem Silicid

$$
\frac{106.3}{108.58}=97.88 \% \text { nichtoxydierte Substanzen. }
$$

Hieraus ergeben sich also $2.12 \%$ koblensaurer Kalk. Diese enthalten 0.85 Tle. Ca. Für das reine Silicid verbleiben noch

$$
44.34-0.85=43.49 \% \text { Ca. }
$$

Unlösliches Eisen sind in dem Silicid

$$
0.95-0.32=0.63 \% \text {. }
$$

Diese entsprechen $0.63 \%$ Si. Von dem Gesamtsilicium sind abzuziehen $2.19 \%$ (freies $\mathrm{Si})+0.63=2.82 \%$. Es verbleiben demnach für das Silicid

$$
52.85-2.82=50.03 \% \text { si. }
$$

Das reine Silicid enthielt auf 43.49 Tle. Ca 50.03 Tle. Si, oder auf $100 \%$ umgerechnet, $53.50 \% \mathrm{Si}$ und $46.50 \% \mathrm{Ca}$. Die Formel $\mathrm{Ca}_{0} \mathrm{Si}_{10}$ verlangt $54.14 \%$ si und $45.86 \% \mathrm{Ca}$. 
Anfänglich wurde das abgesiebte, nicht in Reaktion getretene Silicium von neuem mit Calcium erhitzt, aber die Mischung reagierte viel schwerer. Das entstandene Silicid enthielt mehr Calcium, entwickelte mit Salzsäure ziemlich reichlich selbstentzündlichen Wasserstoff und erwies sich als ein Gemenge von Silicid I und II. Diese erschwerte Reaktion ist jedenfalls auf eine geringe oberfïchliche Oxydation des Siliciums zurückzuführen, wodurch der innige Kontakt der beiden Stoffe verhindert war.

Silicid II. Zur Darstellung dieses Silicids wurden $10 \mathrm{~g}$ geraspeltes Calcium, Calciumgries, mit ca. $3 \mathrm{~g}$ gepulvertem Silicium gemischt, in einem Porzellantiegel fest eingepackt und unter Überleiten von Wasserstoff erwärmt. Die Reaktion verläuft bei niederer Temperatur, so dafs ein Erwärmen mittels Bunsenbrenner genügt. Wenn zur Einleitung der Reaktion stärker erbitzt werden mufs, dann war das Gemisch nicht fein genug. Bei zu hoher Temperatur ist die Reaktion zn heftig, so dafs der Tiegelinhalt herausgeschleudert wird. Die normal verlaufende Reaktion ist von einer Feuererscheinung begleitet und nach dem Erkalten ist die Masse zusammengeschmolzen.

Das Rohprodukt wurde grob gepulvert und mit eiskaltem Zuckerwasser behandelt, wobei das überschüssige Calcium unter Wasserstoffentwickelung in Lösung ging. Sobald die Gasentwickelung aufgehört hatte, wurde abgesaugt, mit kaltem Wasser gewaschen, mit Alkohol und Äther nachgespült und bei $120^{\circ}$ getrocknet.

Die Analyse des Produktes ergab folgende Werte:

$0.1428 \mathrm{~g}$ angew. Substanz ergaben

$$
\begin{aligned}
& 0.1051 \mathrm{~g} \mathrm{SiO}_{2}=34.60 \% \mathrm{si} \\
& 0.1239 \mathrm{~g} \mathrm{CaO}^{2}=62.03 \% \mathrm{Ca} \\
& 0.0029 \mathrm{~g} \mathrm{Fe}_{2} \mathrm{O}_{3}=1.42 \% \mathrm{Fe}
\end{aligned}
$$

$0.1004 \mathrm{~g}$ Silicid entwickelten $83.83 \mathrm{ccm}$ Wasserstoff ron $0^{\circ}$ und $760 \mathrm{~mm}$. $1 \mathrm{~g}$ demnach $0.835 \mathrm{l}$.

Nach den Analysenresultaten soliten entwickelt werden von

$$
\begin{aligned}
& 34.60 \% \text { Si } \quad 54.591 \\
& 62.03 \% \mathrm{Ca} \quad 34.741 \\
& 1.42 \% \mathrm{Fe} \quad 0.57 \mathrm{l} \\
& 89.901 \text { Wasserstoff. }
\end{aligned}
$$


Das Silicid enthielt demnach $\frac{83.50}{89.90}=92.88 \%$ nichtoxydierte Substanz.

Und demnach $7.12 \%$ Verunreinigungen, unter denen auch diesmal etwas Wasser sein kann. Wird die gesamte Menge als Calciumcarbonat angenommen und entsprechend in Rechnung gesetzt, so findet man, dafs von dem Gesamtcalcium $2.85 \%$ abzuziehen sind. Es verbleiben:

$$
62.03-2.85=59.18 \% \mathrm{Ca} .
$$

Von dem Eisen waren $0.32 \%$ in Salzsäure unlöslich, diese entsprechen $0.32 \% \mathrm{Si}$, die von dem Gesamtsilicium abzuziehen sind. Es verbleiben:

$$
34.6-0.32=34.28 \% \mathrm{si} \text {. }
$$

Das reine Silicid enthielt auf 34.28 Tle. Silicium 59.18 Tle. Calcium. Oder in Prozenten:

$$
36.68 \% \text { si und } 63.32 \% \mathrm{Ca} \text {. }
$$

Die Formel $\mathrm{Ca}_{12} \mathrm{Si}_{10}$ verlangt $37.25 \%$ Si und $62.75 \%$ Ca.

Wahrscheinlicher ist, wie später gezeigt werden soll, die Formel $\mathrm{Ca}_{11} \mathrm{Si}_{10}$, die

verlangt.

$$
39.17 \% \text { Si und } 60.83 \% \mathrm{Ca}
$$

Während der Untersuchung dieses Silicids erschien eine Publikation von L. Hackspile, ${ }^{1}$ in der er ein neues Silicid des Calciums von der Formel $\mathrm{Ca}_{3} \mathrm{Si}_{2}$ bescbrieb, das er gelegentlich eines Versuches, wobei Calciumdampf mit Porzellan in Beruhrung kam, beobachtete und ebenfalls durch Erhitzen von Silicium mit überschüssigem Calcium darstellte.

\section{Eigenschaften der silicide.}

Silicid I.

Dieses Silicid ist ein blaugrauer krystallinischer Körper, der bei ca. $1200^{\circ}$ schmilzt. Die Krystalle sind sehr hart und in allen Lösungsmitteln unlöslich. Von den Halogenen wird es beim Erhitzer leicht angegriffen. An der Luft geglüht, verändern sich grobe Krystalle nur wenig, während das fein pulverisierte Silicid leicht verbrennt, wobei ein stickstoffhaltiges Produkt entsteht. Ebenso

${ }^{1}$ Bull. soc. chim. [4] 3 u. 4 (1908), 619. 
verändern Wasser, Säuren und Alkalien das Silicid leicht. Verdünnte Laugen, kohlensaures Alkali lösen das Silicid unter Wasserstoffentwickelung. Konzentrierte Schwefelsäure greift es kaum an. Ebenso konzentrierte Salpetersäure. Die Zersetzung mit Wasser, Essigsäure und Salzsäure beanspruchen besonderes Interesse und sollen daher einzeln beschrieben werden.

Die Zersetzung mit Wasser ist schon ausführlich von Morssan ${ }^{2}$ studiert worden. Er fand, dafs das fein gepulverte Silicid mit Wasser übergossen sich sebr langsam zersetzte ohne Bildung von Silicium wasserstoff.

Das Silicid I wurde ebenfalls von kaltem Wasser kaum angegriffen. Beim Erwärmen dagegen ging Calcium unter Wasserstoffentwickelung in Lösung. Die Gasentwickelung hörte nach kurzer Zeit auf und beim weiteren Kochen trat nur nocb eine mäfsige Entwickelung ein. Folgende Versuchsreihe soll ein Bild dieser Zersetzung geben.

$0.166+\mathrm{g}$ entwickelten beim Kochen

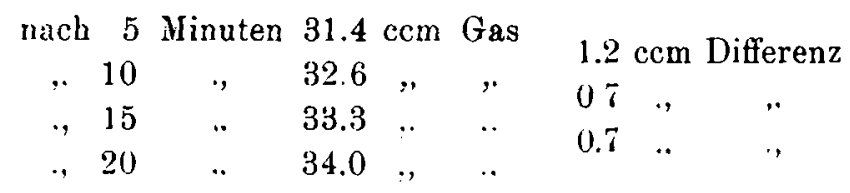

Die nach 5 Minuten erhaltene Wasserstoffmenge ist abhängig von der Menge des Wassers und von den darin enthaltenen Verunreinigungen, wie Kohlensäure. Je mehr Wasser man anwendet desto mehr Gas erhält man und man kann eive gewisse Menge Silicid in kurzer Zeit zersetzen, wenn man es nach jedesmaligem Autkochen mit frischem Wasser zersetzt. Mit einer kleineren Menge Wasser ist das Silicid selbst durch längeres Kochen kaum vollständig zn zersetzen. Aufserdem hängt die Menge des Wasserstoffs von der in dem Wasser gelösten Kohlensäure ab. indem diese stark zersetzend auf das Silicid wirkt. Aus den Versuchen geht hervor, dafs der in Lösung gegangene Kalk hemmend auf die Zersetzung des Silicids einwirkt, indem es nur so lange von dem Wasser zersetzt wird, als dieses noch nicht mit Kalk gesättigt ist. Sobald die Sättigung erreicht ist, hört die Wasserstoffentwickelung auf. Bestätigt wurde diese Ansicht durch einen Versuch mit Kalkwasser. Wurde das Silicid mit Kalkwasser gekocht, so entwickelten sich

Ber. 3อ̄ I, 1108. 
nur ganz geringe Mengen Wasserstoff. Hiermit im Gegensatz befinden sich die Resultate der Versuche Morssans, wonach die Zersetzung des Silicids durch Kalkwasser und Barytwasser beschleunigt wird. Auf welche Weise das Wasser noch zersetzend wirken kann, geht aus folgenden Versuchen hervor. Das reine Silicid, mit Salzsäure gekocht, liefert rein gelbe Silikone. Wird das Silicid mit verdünnter Essigsäure gekocht, so entsteht ein schwarzer krystallinischer Körper, der mit konzentrierter Salzsäure ebenfalls rein gelbe Silikone liefert. Ferner liefert das Silicid mit Wasser gekocht und hierauf mit Salzsäure rein gelbe Silikone. Kocht man aber das Silicid nacheinander mit Wasser, dann mit Essigsäure und zum Schlufs mit Salzsäure, so erhält man einen graugelben Körper, der wahrscheinlich aus einem Gemisch eines scbwarzen und gelben Körpers besteht. Dasselbe tritt ein, wenn man das Silicid erst mit Essigsäure, dann mit Wasser und zum Schlufs mit Salzsäure kocht. Es mufs also durch das Kochen mit Wasser eine weitgehende Zersetzung in dem Silicidmolekül vor sich gegangen sein.

Durch das Kochen mit Wasser treten in das Silicidmolekül die Elemente des Wassers oder auch nur OH-Gruppen ein, diese werden aber zum Teil erst bei sehr hoher Temperatur in Form von Wasser wieder abgespalten.

\section{Zersetzang mit Essigsänre.}

Fssigsäure wirkt schon in ganz verdünnten Lösungen auf das Silicid ein und $z$ war auch schon in der Kälte. Genau wie beim Wasser verläuft die Zersetzung anfangs rasch und nach kurzer Zeit ganz langsam. Folgende Versuchsreihe soll ein Bild dieser Zersetzungsgeschwindigkeit geben. Verwandt wurde doppelt-normale Éssigsäure.

$0.1208 \mathrm{~g}$ entwickelten

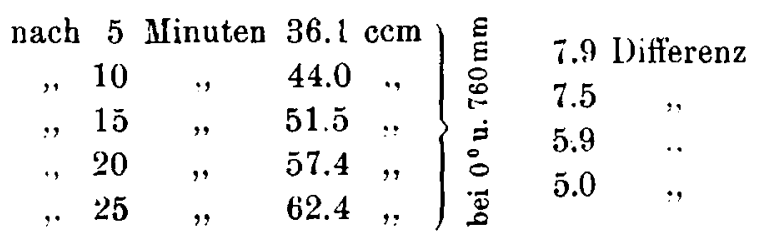

Man sieht, dafs die Zersetzung viel rascher verläuft, wie beim Wasser. Das Silicid wird nach 4-5 stündigem Kochen rollständig zersetzt. 
Das nach der ersten stürmischen Gasentwickelung abtiltrierte Produkt ist schwarz, krystallinisch und ähnlich den Silikonen. Das bei $120^{\circ}$ getrocknete Produkt enthielt $53 \%$ Si und $21 \%$ Ca. Die Substanz verbrannte zu schwer und deshalb konnte eine Wasserstoffbestimmung nicht ausgeführt werden.

Konzentrierte Essigsäure, selbst mit Anhydrid versetzte, also wasserfreie Essigsäure, gibt Wasserstoff und ein Hlockiges Produkt mit $55 \%$ Si und $27 \%$ Ca. Dieser Körper erleidet beim Erhitzen im Kohlensäurestrom bis $200^{\circ}$ keine Veränderung, wird aber durch Kochen mit Wasser vollständig zersetzt.

\section{Zersetzung mit Salzsänre.}

Das Silicid liefert, mit verdünnter Salzsäure übergossen, wenig selbstentzündlichen Wasserstoff, daneben als Endprodukt ein gelblichweilses Silikon. Durch Kochen mit verdünnter Salzsäure tritt eine weitere Zersetzung ein, und nach 4-5 Stunden ist das Silicid vollständig zersetzt. Folgende Versuchsreihe gibt die Zersetzungsgeschwindigkeit wieder. Verwandt wurde doppelt-normale Salzsäure.

$0.0999 \mathrm{~g}$ Silicid lieferten nach

5 Minuten $41.5 \mathrm{ccm}$ Wasserstoff

$\begin{array}{lllllll}10 & , & 50.5 & . & . & 9.0 & \text { Differenz } \\ 15 & , & 56.0 & ., & ., & 5.5 & " \\ 20 & . & 60.3 & ., & . & 4.3 & " \\ 25 & , & 63.7 & . . & ., & 3.4 & ", \\ 30 & , & 66.7 & ., & , . & 3.0 & \end{array}$

Die Zersetzungsgeschwindigkeit ist anfangs gleich der Essigsäure. Sie fällt aber viel rascher ab als jene. Die Silikone baben je nach der Konzentration der Salzsäure oder der Dauer der Einwirkung eine andere Zusammensetzung. Ihre Bestimmung hat also wenig Wert. Konzentrierte Salzsäure wirkt auf das reine Silicid fast ohne Bildung von selbstentzuindlichem Wasserstoff ein. Derselbe entzündet sich höchstens in grölseren Mengen, manchmal auch uberhaupt nicht.

Die Zersetzungsgeschwindigkeit beim Kochen ist die folgende: $0.1037 \mathrm{~g}$ Silicid lieferten

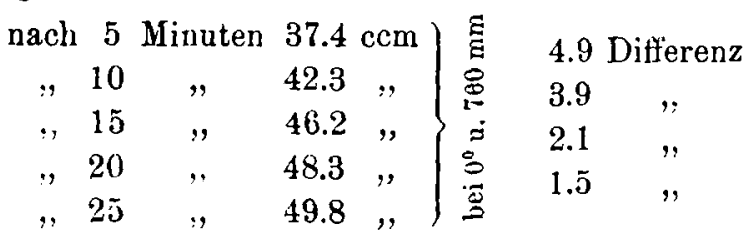


Die Zersetzungsgeschwindigkeit ist also viel geringer als vorher. Je konzentrierter die Salzsäure ist, desto schwerer werden die Silikone zersetzt, was bei ihrer Darstellung von Wichtigkeit ist.

\section{Die Silikone.}

Die Silikone wurden zuerst von WöHLER ${ }^{\perp}$ beschrieben, der sie ebenfalls aus Calciumsilicid darstellte. Er orbielt sie daraus durch Behandeln mit Salzsäure. Es waren orangegelbe krystallinische Körper, die nur von Flufssäure und Alkalien angegriffen wurden. Am Licht zersetzten sie sich unter Wasserstoffentwickelung zu einem weifsen Körper, den er Leukon nannte. Die Analyse des Silikons ergab

$$
67-70 \% \text { Si und } 2.4-2.5 \% \mathrm{H} \text {. }
$$

Daraus berechnete sich die Formel

$$
\mathrm{Si}_{8} \mathrm{H}_{8} \mathrm{O}_{0} \text { oder } \mathrm{Si}_{8} \mathrm{H}_{6} \mathrm{O}_{4} \text {. }
$$

GedThER $^{2}$ erhielt aus Siliciummagnesium, das er aus Kieselfluornatrium und Magnesium darstellte, weilses Silikon, das

$$
42-48 \% \text { Si und } 1.8-2 \% \mathrm{H}
$$

enthielt. Ein ganz reines Produkt ergab die Werte

$$
50.5 \% \mathrm{Si} \text { und } 1.5 \% \mathrm{H} \text {. }
$$

Gecther stellte hierfür die Formel $\mathrm{HSiO}_{5}$ auf.

Vor einigen Jahren veröffentlichte noch Bovdouard" eine Arbeit über diese Körper. Er stellte die Silikone aus Siliciumstahl und Salzsäure dar. Die Analyse ergab

$$
0.88-20.6 \% \mathrm{H}_{2} \mathrm{O}, 1.76-3.24 \% \mathrm{H}, 47.6-50 \% \mathrm{Si} \text {. }
$$

Err betrachtet die Silikone als wasserhaltige ternäre Verbindungen, die sich der von FrLedel und LADENBURG dargestellten Silikoameisensäure und -oxalsäure nähern. Seine Silikone sollten zur Hälfte aus beiden Körpern bestehen.

Die Darstellung und Bestimmung der Silikone aus dem Calciumsilicid geschah in folgender Weise:

1 Ann. Chem. u. Pharm. 127, 257.

a Journ. prakt. Chem. 95 (1865), 425.

${ }^{3}$ Bull, soc. chim. [3] 35 (1906), 710.

Z. anorg. Chem. Bd. 64 . 
Eine abgewogene Menge pulverisierten Silicids wurde in einem Erlenmeyer im Kohlensäurestrom mit konzentrierter Salzsäure übergossen und möglichst unter Lichtabschlufs kurz aufgekocht. Die Reaktion wurde deswegen im Kohlensäurestrom vorgenommen, weil durch Verbrennen von Siliciumwasserstoff leicht etwas Kieselsäure den Silikonen beigemengt werden konnte. Hierauf wurde die Flüssigkeit abgekühlt, mit Wasser verdünnt und in einem gewogenen Goochtiegel abfiltriert. Die Silikone wurden mit kaltem, salzsäurehaltigem Wasser gewaschen, mit Alkohol und Äther getrocknet und gewogen. Im Filtrat wurde das Calcium bestimmt. Ebenso in einer anderen Probe die entwickelte Wasserstoffmenge. Die Silikone wurden hierauf noch einmal bei $120^{\circ} \mathrm{im}$ Kohlensäurestrom getrocknet und wieder gewogen.

Die Analyse wurde auf folgende Weise ausgeführt: Die Silikone wurden durch Kochen mit Natronlauge zersetzt, die entwickelte Wasserstoffmenge bestimmt und in der zurückbleibenden Lösung auf die gewöbnliche Art das Silicium. In einer anderen Probe wurde durch Verbrennen im Sauerstoffstrom in Gegenwart von Kupferoxyd der Wasserstoff bestimmt. Die Silikone sind im trockenen Zustande sehr unbeständig und müssen rasch verarbeitet werden.

1. Bestimmungen der Ausbeute an Silikon.

Angew. Silicid $1.3982 \mathrm{~g}$.

Daraus wurden folgende Mengen Silikon erhalten:

bei gewöhnlicher Temperatur getrocknet $1.2002 \mathrm{~g}-85.84 \%$ bei $120^{\circ}$ getrocknet . . . . . . . $1.1116 \mathrm{~g}=79.50 \%$

Im Filtrat fanden sich:

$$
\text { Verlust beim Trocknen } 6.34 \%
$$

$$
\begin{aligned}
& 0.0090 \mathrm{~g} \mathrm{Fe}_{2} \mathrm{O}_{3}=0.45 \% \mathrm{Fe} \\
& 0.8710 \mathrm{~g} \mathrm{CaO}^{2}=44.54 \% \mathrm{Ca} .
\end{aligned}
$$

$0.1024 \mathrm{~g}$ Silicid ergaben $28.04 \mathrm{ccm}$ Wasserstoff von $0^{\circ}$ und $760 \mathrm{~mm}$.

2. Die Analyse des Silikons ergab. $0.1218 \mathrm{~g}$ angew. Substanz lieferten:

$$
\begin{aligned}
& 0.1722 \text { g } \mathrm{SiO}_{2}=66.48 \% \mathrm{Si} \\
& 0.0090 \mathrm{~g} \mathrm{Fe}_{2} \mathrm{O}_{3}=0.52 \% \mathrm{Fe}
\end{aligned}
$$

und $104.6 \mathrm{ccm}$ Wasserstoff von $0^{\circ}$ und $760 \mathrm{~mm}=7.74 \%$. Die Wasserstoff bestimmung bei der Verbrennung ergab: $0.2753 \mathrm{~g}$ angew. Substanz lieferten:

$$
0.0689 \mathrm{~g} \mathrm{H}_{2} \mathrm{O}=2.80 \% \mathrm{H} \text {. }
$$


3. Berechnung der Formel; das ursprüngliche Silicid enthielt:

$$
52.85 \% \mathrm{Si}, 0.95 \% \mathrm{Fe}, 44.34 \% \mathrm{Ca} \text {. }
$$

Berechnet man hieraus und aus dem oben angegebenen Wert für die Ausbeute an Silikon, wieviel Silicium in dem Silikon enthalten sein mülste, wenn kein Verlust an Silicium als Siliciumwasserstoff stattgefunden hat, so findet man folgenden Wert:

$$
\frac{79.5}{\overline{0} 2 . \overline{5}}=\stackrel{100}{x} \quad x=66.47 \% \mathrm{Si} .
$$

Das ganze Silicium findet sich also in dem Silikon wieder, und es ist kein Silicium als Siliciumwasserstoff verloren gegangen.

Im Filtrat befanden sich $0.45 \%$ Fe und $44.54 \%$ Ca. $100 \mathrm{~g}$ Silicid verloren danach $44.99 \% \mathrm{Fe}+\mathrm{Ca}$.

Aus der Bestimmung der Ausbeute an Silikon geht aber hervor, dafs $100 \mathrm{~g}$ Silicid nur $20.5 \mathrm{~g}$ verloren. Es mufsten demnach $44.99-20.5=24.49 \mathrm{~g}$ Wasserstoff und Sauerstoff aufgenommen worden sein, und es mülsten danach in den Silikonen davon enthalten sein:

$$
\frac{24.49}{7 \overline{9} .50}=30.8 \% \text {. }
$$

Die Differenz von 100 aus der Analyse des Silikons ergibt $33.0 \%$ Wasserstoff und Sauerstoff.

Das Silikon enthält unter Ausschaltung des Eisens:

$$
66.83 \% \mathrm{Si}, 2.81 \% \text { H und } 30.36 \% \mathrm{O} \text {. }
$$

Die Formel $\mathrm{Si}_{10} \mathrm{H}_{13} \mathrm{O}_{8}$ verlangt:

$$
66.96 \% \mathrm{Si}, 2.86 \% \mathrm{H} \text { und } 30.18 \% \mathrm{O} \text {. }
$$

Wöhler fand, wie schon angegeben:

$$
67-70 \% \mathrm{Si}, 2.4-2.5 \% \mathrm{H} \text {. }
$$

Yach diesen Zahlen würde auch noch die Formel $\mathrm{Si}_{10} \mathrm{H}_{10} \mathrm{O}_{8}$ in Betracht kommen, die einer später angegebenen Konstitutionsformel besser entspricht und $67.28 \% \mathrm{Si}, 2.38 \% \mathrm{H}$ und $30.34 \mathrm{O}$ verlangt.

Das Silikon erfordert nach der Gleichung:

$$
\begin{aligned}
& \mathrm{Si}_{10} \mathrm{H}_{18} \mathrm{O}_{8}+12 \mathrm{H}_{2} \mathrm{O}=10 \mathrm{SiO}_{2}+36 \mathrm{H} \text { oder } \\
& \mathrm{Si}_{10} \mathrm{H}_{10} \mathrm{O}_{8}+12 \mathrm{H}_{8} \mathrm{O}=10 \mathrm{SiO}_{2}+34 \mathrm{H}
\end{aligned}
$$

eine Gasentwickelung von 8.57 bzw. $8.11 \%$ H. Gefunden wurde $7.74 \% \mathrm{H}$. 


\section{Eigenschaften des Silikons I.}

Das Silikon ist ein orangegelber bis bellgelber krystallinischer unlöslicher Körper, leicht zersetzlich und zerfällt schon im Lichte unter Bildung eines weilsen Körpers, des Leukons. Bei Gegenwart von Wasserstoffionen ist es ziemlich bestäudig, dagegen Hydroxylionen gegenüber sehr empfindlich und wird demgemüls von Süuren kaum angegriffen, dagegen leicht zerstört durch Kochen mit Wasser, Natronlauge, kohlensaurem Alkali usw. In der Kälte tritt keine vollständige Zersetzung ein, bei Ammoniak selbst beim Kochen nicht. Fs bildet sich bier neben Kieselsäure ein rotbrauner Körper, der, mit Natronlauge gekocht, noch Wasserstoff abspaltet.

\section{Eigenschaften des Silicids II.}

Dieses Silicid bildet nach dem Fortlösen des überschüssigen Calciums ein krystallinisches unlösliches Pulver, das sich leicht durch Kochen mit Wasser zersetzt; selbst Kalkwasser bewirkt hier eine leichte Zersetzung.

Das Silicid entwickelt beim Übergiefsen mit verdünnter Essigsäure Wasserstoff, der an einer Flamme explodiert. Mit verdünnter oder konzentrierter Salzsäure entstehen dagegen reichliche Mengen von selbstentzündlichem Wasserstoff und aulserdem ein weifses oder schwachgelbes Silikon, das am Licht zum Teil in einen gelben und scblielislich wieder in einen weirsen Körper übergeht. Auch $\mathrm{H}_{\text {ACK- }}$ spul erhielt aus seinem Silicid $\mathrm{Ca}_{3} \mathrm{Si}_{2}$ ein weifses Silikon. Konzentrierte Salpetersäure wird reduziert.

\section{Darstellung und Eigenschaften des Silikons II.}

Die Darstellung und Analyse geschah in derselben Weise wie bei Silikon $I$.

$1.0444 \mathrm{~g}$ Silicid ergaben:

bei gewöhnlicher Temperatur getrocknet $0.6996 \mathrm{~g}-66.98^{\circ} \%$

bei $120^{\circ}$ getrocknet . . . . . . . $0.6188 \mathrm{~g}-59.25 \%$

Das Filtrat enthielt:

$$
\text { Verlust beim Trocknen } 7.73 \%
$$

$$
\begin{aligned}
& 0.0119 \mathrm{~g} \mathrm{Fe}_{2} \mathrm{O}_{3}=0.80 \% \mathrm{Fe} \\
& 0.9036 \mathrm{~g} \mathrm{CaO}=61.86 \% \mathrm{Ca} .
\end{aligned}
$$

$0.1406 \mathrm{~g}$ entwickelten $90.98 \mathrm{ccm}$ Wasserstoff von $0^{\circ}$ und $760 \mathrm{~mm}$, $1 \mathrm{~g}$ demnach $0.647 \mathrm{l} \mathrm{H}$. 
Die Analyse des Silikons lieferte folgende Werte. $0.1617 \mathrm{~g}$ angew. Substanz ergaben:

$$
\begin{aligned}
& 0.1742 \mathrm{~g} \mathrm{SiO}_{2}=50.65 \% \mathrm{Si} \\
& 0.0014 \mathrm{~g} \mathrm{Fe}_{2} \mathrm{O}_{3}=0.61 \% \mathrm{Fe}
\end{aligned}
$$

daneben $50.04 \mathrm{ccm}$ Wasserstoff von $0^{0}$ und $760 \mathrm{~mm}=2.79 \% \mathrm{H}$.

Die Wasserstoff bestimmung durch Verbrennung ergab für $0.2939 \mathrm{~g}$ angew. Substanz:

$$
0.0409 \mathrm{~g} \mathrm{H}_{2} \mathrm{O}=1.56 \% \mathrm{H} \text {. }
$$

Das ursprüngliche Silicid enthielt $34.60 \% \mathrm{Si}, 62.03 \% \mathrm{Ca}$ und $1.42 \%$ Fe. Berechnet man hieraus, wie bei Silicid I, wieviel Silicium in dem Silikon vorhanden sein mülste, wenn kein Verlust an Siliciumwasserstoff stattgefunden hätte, so findet man nach der Gleichung:

$$
\frac{59.25}{34 . \overline{60}}=\frac{100}{x} \quad x=58.40 \mathrm{Si} .
$$

Gefunden wurden $50.65 \%$ Si. Es sind demnach $7.75 \%$ als Siliciumwasserstoff verloren gegangen.

Das ursprüngliche Silicid verlor $61.86 \% \mathrm{Ca}, 0.80 \% \mathrm{Fe}$ und $7.75 \% \mathrm{Si}$, zusammen $70.41 \%$. In Wirklichkeit verlor es aber nur

$$
(100-59.25)=40.75 \% \text {. }
$$

Die Differenz $(70.41-40.75)=29.66$ oder $50.06 \%$ vom Gewicht des Silikons entspricht dem aufgenommenen Wasserstoff und Sauerstoff. Aus der Analyse ergibt sich ein Gehalt von

$$
(100-[50.6 \overline{0}+0.61])=48.74 \% \text {. }
$$

Unter Ausschaltung des Eisens ergibt sich für das Silikon:

$$
50.96 \% \mathrm{Si}, \quad 1.57 \% \mathrm{H} \text { und } 47.47 \% \mathrm{O} .
$$

Die Formel $\mathrm{Si}_{8} \mathrm{H}_{7} \mathrm{O}_{13}$ bzw. $\mathrm{Si}_{8} \mathrm{H}_{8} \mathrm{O}_{13}$ verlangt:

$$
\begin{aligned}
& 51.37 \% \mathrm{Si}, 1.60 \% \mathrm{H} \text { und } 47.03 \% \mathrm{O} \text {. } \\
& 51.25 \% \mathrm{Si}, 1.819 \% \mathrm{H} \text { und } 46.92 \% \mathrm{O} \text {. }
\end{aligned}
$$

Das Silikon erfordert beim Lösen in Natronlauge nach der Gleichung:

$$
\begin{aligned}
& \mathrm{Si}_{8} \mathrm{H}_{7} \mathrm{O}_{13}+3 \mathrm{H}_{2} \mathrm{O}=8 \mathrm{SiO}_{2}+13 \mathrm{H} \\
& \mathrm{Si}_{8} \mathrm{H}_{5} \mathrm{O}_{23}+3 \mathrm{H}_{2} \mathrm{O}=8 \mathrm{SiO}_{2}+14 \mathrm{H}
\end{aligned}
$$


eine Gasentwickelung von $2.96 \%$ bzw. $3.18 \%$ Wasserstoff. Gefunden wurden $2.79 \%$.

Aus dieser Silikonbildung geht hervor, dals in dem ursprünglichen Silicid mindestens 8 Siliciumatome vorbandeu sein mulsten, wenn man bei der Reaktion mit Salzsäure keine Verkettung ron Siliciumatomen als solche oder durch Vermittelung von Sauerstoff annebmen will. Unter Berücksichtigung des Verlustes an Silicium als Siliciumwasserstoff findet man für das Silicid die wabrscheinlichste Formel $\mathrm{Ca}_{11} \mathrm{Si}_{10}$. Die Bildung des Silikons aus dem Silicid erfolgt alsdann nach der Gleichung:

$$
\begin{aligned}
& \mathrm{Ca}_{11} \mathrm{Si}_{10}+24 \mathrm{H}_{2} \mathrm{O}=\mathrm{Si}_{8} \mathrm{H}_{7} \mathrm{O}_{13}+2 \mathrm{SiH}_{4}+33 \mathrm{H}+11 \mathrm{CaO} \text { oder } \\
& \mathrm{Ca}_{11} \mathrm{Si}_{10}+24 \mathrm{H}_{2} \mathrm{O}=\mathrm{Si}_{9} \mathrm{H}_{8} \mathrm{O}_{13}+2 \mathrm{SiH}_{4}+32 \mathrm{H}+11 \mathrm{CaO} .
\end{aligned}
$$

In seinen übrigen Eigenschaften unterscheidet sich das Silikon nicht weiter vom Silikon I.

Es darf wohl zweifellos angenommen werden, dafs die silikone keine einfach zusammengesetzten Körper sind, was schou aus der Konstitution der Silikoameisensäure und Silikooxalsäure zu entnehmen ist. Dann ist aber auch die Zusammensetzung der Silicide keine so einfache wie die des Calciumcarbids, weil sonst die Bildung der Silikone erst durch verschiedene Kondensationsvorgünge erfolgen könnte.

\section{Einwirkung von Stickstoff auf die Silicide.}

Im Jahre 1865 hat Geuther ${ }^{2}$ das Verbalten des Calcium- und Magnesiumsilicids gegen Stickstoff geprüf, indem er beide Silicide in Stickstoffstrome auf starke Hellrotglut erhitate. Das Calciumsilicid hatte um 5.2\% zugenommen, aber nur die Oberfläche war verändert und hatte ein dunkleres Aussehen angenommen. Beim Schmelzen mit Kali entwickelte es Ammoniak. Das Maguesiumsilicid lieferte bei gleicher Behandlung eine schwarze Masse, die sich wie ein Gemenge von Silicium und Magnesiumnitrid verhielt. Geuther erklärt ausdrücklich, dafs in beiden Fällen nicht die Bildung eines Stickstoffsiliciummetalles stattfand, sondern nur die Bildung von Stickstoffmetall unter Entbindung des Siliciums. Die folgenden Versuche lassen aber das aufserordentliche Aufnahmerermögen des Calciumsilicids für Stickstoff erkennen unter Bildung neuer sehr stabiler Körper. Die Versuchsanordnung war folgende: Die Fr-

1 Journ. prukt. Chen. $95,425$. 
wärmung des Silicids geschah in einer Porzellanröhre, die in einem elektrischen Widerstandsofen erbitzt wurde. Die Temperaturmessung erfolgte mit Hilfe eines Millivoltmeters und Thermoelementes, das aufsen um die Porzellanröhre gewickelt war. Der Stickstoff wurde in üblicher Weise aus Ammonsulfat, Natriumnitrit und etwas Bicbromat bereitet und mit alkalischem f'errosulfat, sowie Pyrogallol gewaschen. For dem Überleiten über das Silicid wurde derselbe nochmals durch alkalische Pyrogalluslösung geleitet, dann mit Schwefelsäure und Chlorcalcium getrocknet.

Das Silicid befand sich in einem Schiffichen, das ror und nach der Absorption gewogen wurde. Am anderen Ende der Porzellanröhre waren $z$ wei entgegengesetzt miteinander verbundene Waschflaschen angeschlossen, die ein Zurücksteigen des abschliefsenden Wassers in die Röhre bei zu heftiger Absorption verhüten sollten.

Wurde auf diese Weise das fein gepulverte Silicid I erhitzt, so trat bei $1010^{\circ}$ eine starke Stickstoffabsorption ein. Die Temperatur stieg plötzlich um $80-100^{\circ}$. Die Absorption war eine sehr energische und vollzog sich innerhalb einer kurzen Spanne Zeit. Erhitzte man weiter, so trat bei $1250-1300^{\circ}$ eine nochmalige aber ganz allmähliche Absorption ein. Das nach der ersten Absorption erhaltene Produkt war zusammengeschmolzen oder auch nur gesintert und hatte gewöhnlich um $22-26 \%$ zugenommen. OberHächlich war die Masse mit einem weil'sen Pulver bedeckt. In der Masse befanden sich oft Krystalle bzw. deren Bruchflächen vom zusammengeschmolzenen unverändertem Silicid. Auch gab das Produkt mit Salzsäure immer noch Silikone. Das auf höhere Temperatur erhitzte Produkt war dagegen vollständig verändert. Es war eine graue zusammengesinterte Masse, die sich mit Salzsäure äufserlich nicht mehr veränderte. Dasselbe Produkt konnte auch leicht bei niederer Temperatur erhalten werden, wenn man das nach der ersten Reaktion erhaltene nur teilweise umgewandelte Produkt fein pulverte und im Stickstoffstrom noch einmal erhitzte. Da das stickstofthaltige Produkt unschmelzbar ist, so verhinderte dieses jetzt das Zusammenschmelzen der Masse, und sie konnte also vollständig mit dem Stickstoff in Reaktion treten. Bei dem zweiten Male trat genau bei derselben Temperatur wieder die Absorption ein, nur war sie jetzt naturgemäfs viel schwächer. Das Produkt hatte noch einmal um 8-12\% zugenommen und war fast weifs geworden. Beim dritten Erhitzen wurden nur noch $0.5-1 \% \mathrm{~N}$ aufgenommen. Das auf diese Weise erhaltene fast weifse Pulver roch schwach 
nach Ammoniak. Durch Kochen mit Wasser zersetzte es sich sehr langsam unter Ammoniakabspaltung. Etwas rascher ging die Zersetzung mit verdünnter Natronlange vor sich. Wurde das Pulrer mit konzentrierter Salzsäure gekocht, so trat äufserlich keine Veränderung ein. Es ging jedoch ein Teil des Calciums in Lösung und ebenso wurde ein Teil des Stickstoffs als Ammoniak abgespalten. Bei längerem Kochen mit Salzsäure wurde die Masse schwammiger. Die grölste Ammoniakmenge wurde erhalten, wenn man das Produkt erst mit Salzsäure kochte, hierauf mit Natronlauge übersättigte, wobei sich etwal Wasserstoff entwickelte, und dann das Ammoniak abdestillierte. Beide Reagenzien allein angewandt ergaben eine geringere Ammoniakmenge. Vollständig liefs sich der Stickstoff nur durch Schmelzen des Körpers mit Kalihydrat abspalten. Man sieht also, dafs sich das Produkt fast wie Siliciumstickstoff verhält.

Silicid II nahm beim Erhitzen im Stickstuffstrome von 950 bis $960^{\circ}$ an Stickstoff auf, bei $1000^{\circ}$ wurde auch hier die Reaktion lebhafter, erreichte jedoch nicht den Grad wie bei Silicid I. Die Absorption hatte auch bald ihr Ende erreicht. Das Produkt hatte um 12-13\% zugenommen und war nicht zusammengeschmolzen. Bei dem zweiten und dritten Erhitzen nahm das Produkt jedesmal noch um 2-3\% zu, so dafs also noch eine ganz allmähliche Stickstoffaufnahme stattfand. Das so erhaltene Produkt war schwarz. grau mit einem grünlichen Strich; es roch anfangs stark nach Ammoniak; Wasser und Kalilauge bewirkten langsame Zersetzung; Salzsäure dagegen eine vollständige. Dabei bildeten sich grüngelbe Silikone. Der gesamte Stickstoff und das Calcium wurde abgespalten, ersterer in Form von Ammoniak.

\section{Analyse der Produkte.}

Silicid I. Dieses Silicid nahm bei der ersten Absorption um $24.2 \%$ zu. Das letztere Produkt nahm bei der zweiten Absorption nochmals um $12.3 \%$, zu und bei der dritten Absorption noch um $0.8 \%$.

Das Endprodukt enthieit danach $27.6 \%$ Stickstoff.

Die Gesamtstickstoffbestimmung als Ammoniak durch Schmelzen mit Alkali gelang in dem folgenden kleinen Apparat ziemlich einfach. Das Produkt wurde in ein grölseres Reagenzglas eingewogen und festes Natronhydrat zugegeben. Das Glas wurde mit einem doppelt durchbohrten Gummistopfen versehen, durch dessen eine Bohrung ein fast bis auf den Boden des Reagenzglases reichendes 
Glasrohr ging und das mit einer Waschtlasche mit konzentrierter Schwefelsäure in Verbindung stand. Durch die andere Bohrung führte ein gleich am Stopfen endendes Glasrohr, das unten zugeschmolzen war und nur mit einer kleinen seitlichen Öffnung versehen war. Das andere Ende des Rohres war nach abwärts gebogen und zu einer feinen Capillare ausgezogen. Aufserdem war das Rohr zum Teil mit Glaswolle gefüllt, um mitgerissenes Natronhydrat zurückzuhalten. Dieser Teil des Glasrohres war derart gebogen, dafs das sich dort kondensierte Wasser in das Reagenzglas zurückflofs. Die Capillare tauchte in eine gemessene Menge norm. Schwefelsäure, die sich in einem Ërlenmeyer befand. Dieser wurde mit einer Luftpumpe schwach erakuiert und so ein langsamer Luftstrom durch die ganze Vorrichtung gesaugt. Das Reagenzglas, das zweckmälsig etwas schräg stand, wurde zunächst in seiner ganzen Länge schwach erwärmt, damit sich in dem oberen Teil kein Wasser kondensierte. Hierauf wurde das Natronhydrat vorsichtig geschmolzen, wobei sich das Produkt unter Ammoniakentwickelung zersetzte. Sobald die Gasentwickelung vorüber war, wurde noch kurze Zeit Luft durch den Apparat gesaugt und dann das Reagenzglas durch ein anderes, das mit Wasser gefüllt war, ersetzt. Durch rorsichtiges Kochen des letzteren wurden die in der Röhre allenfalls verbleibenden letzten Reste Ammoniak übergetrieben.

Angew. Substanz $0.3540 \mathrm{~g}$ :

\begin{tabular}{lll} 
vorgelegt & $20 \mathrm{ccm}$ norm. Schwefelsäure \\
zurücktitriert & $13.25, ., \quad, \quad$, \\
\cline { 2 - 3 } & $6.75 \mathrm{~cm}$ norm. Schwefelsäure
\end{tabular}

Diese entsprechen $0.0947 \mathrm{~g}$ Stickstoff oder $26.73 \%$.

Nach 1 stündigem Kochen mit Wasser wurden ca. $2 \%$ Stickstoff als Ammoniak abgespalten. Mit verdünnter Natronlauge etwa $7 \%$. Wurde die Substanz zuerst mit Salzsäure gekocht, dann mit Natronlauge übersättigt und das Ammoniak übergetrieben, so ergab sich folgender Wert.

Angew. Substanz 0.4992 g:

\begin{tabular}{|c|c|c|c|}
\hline rorge & 20.0 & ccm norm. & Schwefelsäure \\
\hline zurücktitriert & 15.23 & , & $"$ \\
\hline
\end{tabular}

Diese entsprechen $0.0669 \mathrm{~g}$ Stickstoff oder $13.41 \%$. Es wurden also hierbei fast genau die Hälfte des Stickstoffs 
abgespalten, während die andere Hälfte im Rückstand rerblieb. Dieser Rückstand wurde nach dem Behandeln mit Natronlauge auf einem Goochtiegel abgesangt, mit Wasser und hierauf mit Salzsäure ausgewaschen, getrocknet und gewogen. Es verblieben:

$$
0.2012 \mathrm{~g}=40.3 \% \text {. }
$$

Der so erhaltene Körper war ein graues, unschmelzbares und unlösliches Pulver, das sich mit Salzsäure und Natronlauge nur sehr langsam zersetzte. Es verhält sich genau wie Siliciumstickstoff.

Die Analyse des Körpers ergab folgende Werte. Angew. Substanz $0.2352 \mathrm{~g}$ gefunden:

$$
\begin{aligned}
& 0.2047 \mathrm{~g} \mathrm{SiO}_{2}=40.92 \% \mathrm{Si} \\
& 0.0883 \mathrm{~g} \mathrm{CaO}^{2}=26.85 \% \mathrm{Ca} .
\end{aligned}
$$

\begin{tabular}{|c|c|}
\hline $\begin{array}{l}\text { vorgelegt } \\
\text { zurücktitriert }\end{array}$ & $\begin{array}{l}20.0 \mathrm{ccm} \\
17.45 \quad \text { norm. Schwefelsäure }\end{array}$ \\
\hline & 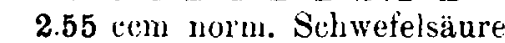 \\
\hline
\end{tabular}

1)ie Stickstutfibestimmung durch Schmelzen mit Alkali ergab) $0.115 \tilde{\mathrm{g}}$ angew. Substanz:

Diese entsprechen $0.03572 \mathrm{~g} \mathrm{~N}$ oder $30.92 \%$.

Berechnet wurde aus dem nicht abspaltbaren Stickstoff der Ursubstan\% folgender Wert. Die Substanz enthielt $26.73^{\circ}{ }_{0}$ Gesamtstickstoff. Mit Salzsäure und Natronlauge waren $13.41 \%_{0}^{\circ}$ abspaltbar. Die Differenz beträgt $13.32 \%$. In $40.3 \mathrm{~g}$ siud demnach $13.32 \mathrm{~g}$ Stickstoff oder $33.05 \%$. Der Körper hatte also die Zusammensetzung:

$$
40.92 \% \mathrm{Si} \quad 26.85 \% \mathrm{Ca} \quad 30.92 \% \mathrm{~N} .
$$

Die Formel $\mathrm{CaSi}_{2} \mathrm{~N}_{3}$ verlangt:

$$
\underline{40.86 \% \mathrm{Si} \quad 28.84 \% \mathrm{Ca} \quad 30.30 \% \mathrm{~N} .}
$$

Die Analyse des ursprünglichen Produkts ergab angew. Sub$\operatorname{stanz} 0.2352 \mathrm{~g}$ :

$$
\begin{aligned}
& 0.1960 \mathrm{~g} \mathrm{SiO}_{2}=39.18 \% \mathrm{Si} \\
& 0.1078 \mathrm{~g} \mathrm{Ca}=32.77 \% \mathrm{Ca} \\
& 0.0021 \mathrm{~g} \mathrm{Fe}_{2} \mathrm{O}_{2}=0.62 \% \mathrm{Fe} .
\end{aligned}
$$

$0.3075 \mathrm{~g}$ entwickelten mit Salzsäure und Natronlauge gekocht $25.57 \mathrm{~cm}$ Wasserstoff von $0^{0}$ und $760 \mathrm{~mm}$ oder $0.742 \%$. wobei sich aber nur mit Natronlauge Wasserstofi' entwickelte.

Für den zersetzbaren Anteil des stickstoffhaltigen Produktes verbleiben demnach: 


$$
\begin{aligned}
& 39.18-(0.403 \cdot 40.92)=22.69 \% \mathrm{Si} \\
& 32.77-(0.403 \cdot 26.85)=21.96 \% \mathrm{Ca} \\
& 26.73-(0.403 \cdot 30.92)=\frac{14.23 \% \mathrm{~N}}{58.88 \%}
\end{aligned}
$$

58.88 Tle. des Produktes enthalten also 22.69 Tle. Silicium, 21.96 Tle. Calcium und 14.23 Tle. Stickstoff; oder in Prozenten

$$
38.54 \% \text { Si, } \quad 37.29 \% \text { Ca und } 24.17 \% \mathrm{~N} \text {. }
$$

Beachtet man, dafs in dem ursprünglichen Silicid $0.85 \%$ Calcium in Oxydform vorhanden war, so erhält man

$$
38.95 \% \mathrm{si}, \quad 36.62 \% \mathrm{Ca} \text { und } 24.43 \% \mathrm{~N} \text {. }
$$

Die Formel $\mathrm{Ca}_{2} \mathrm{Si}_{3} \mathrm{~N}_{4}$ verlangt:

$$
38.45 \% \mathrm{Si}, \quad 36.20 \% \text { Ca und } 25.35 \% \mathrm{~N} \text {. }
$$

Die Reaktion im Stickstoffstrom verläuft demnach nach der Gleichung:

$$
\mathrm{Ca}_{6} \mathrm{Si}_{10}+14 \mathrm{~N}=2 \mathrm{CaSi}_{2} \mathrm{~N}_{3}+2 \mathrm{Ca}_{2} \mathrm{Si}_{3} \mathrm{~N}_{4} \text {. }
$$

In dem letzten Gemisch sollten nach der Gleichung 38.54\% $\mathrm{CaSi}_{2} \mathrm{~N}_{3}$ vorhanden sein. Gefunden wurden $40.3 \%$.

Silicid II nahm bei der ersten Absorption um $12.42 \%$ zu; dieses Produkt bei der zweiten Absorption um $2.86 \%$ und das letzte bei der dritten um $2.37 \%$.

In dem Endprodukt waren demnach etwa $15.02{ }_{0}^{0} \mathrm{~N}$.

Die Gesamtstickstoff bestimmung durch Schmelzen mit Alkali lieferte folgenden Wert. Angew. Substanz 0.2128 g:

vorgelegt $\quad 20.0 \mathrm{ccm}$ norm. Schwefelsäure

zurücktitriert $17.75,, \quad$, ,

verbraucht $2.25 \mathrm{ccm}$ norm. Schwefelsäure

Diese entsprechen $0.0316 \mathrm{~g} \mathrm{~N}$ oder $14.84 \%$.

Nach 1 stündigem Kochen mit Wasser wurden ca. $4.5 \%_{\text {: }}^{\circ}$ Stickstoff als Ammoniak abgespalten. Mit verdünnter Natronlauge aber ca. $6 \%$. Wurde die Substanz erst mit Salzsäure gekocht und dann mit Natronlauge übersättigt und das Ammoniak übergetrieben, so ergab sich; angew. Substanz $0.2506 \mathrm{~g}$ :

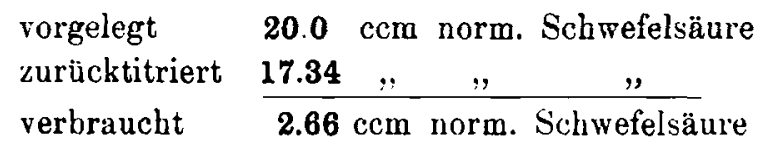


Diese entsprechen $0.03735 \% \mathrm{~N}$ oder $14.9 \%$.

Hier wird also der gesamte Stickstoff mit Salzsäure abgespalten.

Die Analyse des Produktes ergab angew. Substanz $0.2382 \mathrm{~g}$ :

$$
\begin{aligned}
& 0.1487 \mathrm{~g} \mathrm{SiO}_{2}=29.35 \% \mathrm{Si} \\
& 0.1730 \mathrm{~g} \mathrm{Ca}^{2}=51.92 \% \mathrm{Ca} \\
& 0.0040 \mathrm{~g} \mathrm{Fe}_{2} \mathrm{O}_{3}=1.17 \% \mathrm{Fe}
\end{aligned}
$$

$0.1938 \mathrm{~g}$ Substanz entwickelten $58.96 \mathrm{ccm}$ Wisserstoff von $0^{0}$ und $760 \mathrm{~mm}$ oder $2.74 \% \mathrm{H}$.

Von dem ursprünglichen Silicid waren $2.85{ }^{\circ}{ }_{10} \mathrm{Ca}$ in Oxydform vorhanden. Zieht man diese mit in Rechnung, so findet man für das stickstoff haltige Produkt die Werte:

$$
31.33 \% \text { Si. } \quad 52.83 \% \text { Ca und } 15.84 \% \mathrm{~N} .
$$

Die formel $\mathrm{Ca}_{12} \mathrm{Si}_{10} \mathrm{~N}_{11}$, verlangt:

$$
31.37 \% \mathrm{Si}, \quad 53.16 \% \mathrm{Ca} \text { und } 15.47 \% \mathrm{~N} \text {. }
$$

I)ie wahrscheinliche Formel $\mathrm{Ca}_{11} \mathrm{Si}_{10} \mathrm{~N}_{10}$ verlangt:

$$
32.83 \% \mathrm{Si}, \quad 50.99 \% \text { Ca und } 16.18 \% \mathrm{~N} \text {. }
$$

Die Reaktion zwischen dem Silicid und Stickstoff würde d:ını nach folgender Gleichung verlaufen:

$$
\mathrm{Ca}_{11} \mathrm{Si}_{10}+10 \mathrm{~N}=\mathrm{Ca}_{11} \mathrm{~N}_{10} \mathrm{Si}_{10} .
$$

Mit Salısäure gab dieses Produkt neben etwas Wasserstoff schlammiges, gelbgrünes Silikon.

$1 \mathrm{~g}$ Substanz mit Salzsäure gekocht entwickelten $118.8 \mathrm{ccm}$ Wasserstoff ron $0^{\circ}$ und $760 \mathrm{~mm}$ oder $1.06 \%$. Wurden die Silikone in derselben Flüssigkeit mit Natronlauge weiter gekocht, so entwickelten sich noch $185.8 \mathrm{ccm}$ Wasserstoff oder $1.68 \%$. Die Bestimmung des Silikons war sehr schwer, da es sich nicht abfiltrieren liefs. Im Augenblick war jedes Filter verstopft und es lief keine Flüssigkeit mehr durch. Wegen dieser Beschaffenheit des Silikons war auch ein genügendes Auswaschen nicht möglich, da es hartnäckig die Flüssigkeit zurückhielt. Es fanden sich deswegen immer in dem Silikon etwas Calcium und Stickstoff. Die Analyse ergab unter Eliminierung des Calciums und Stickstoffs:

$57.0 \% \mathrm{Si}, 1.15 \% \mathrm{H}$ durch Verbrennung), $3.98 \% \mathrm{H}$ (vol.).

Die Formei $\mathrm{H}_{6} \mathrm{Si}_{10} \mathrm{O}_{13}$ verlangt:

$57.0 \% \%_{10} \mathrm{Si}, 1.22 \%$ H (durch Verbrennung), $4.06 \%$ H (rol.). 
Die Formel ist demnach ähnlich der des Silikons aus Silicid II. Die Bildung des Silikons verläuft nach der Gleichung:

$$
\mathrm{Si}_{10} \mathrm{Ca}_{11} \mathrm{~N}_{10}+24 \mathrm{H}_{2} \mathrm{O}=\mathrm{H}_{6} \mathrm{Si}_{10} \mathrm{O}_{13}+10 \mathrm{NH}_{3}+11 \mathrm{CaO}+12 \mathrm{H} \text {. }
$$

Diese Gleichung erfordert eine Gasentwickelung von $1.40 \%$. Gefunden wurden $1.06 \%$.

\section{Konstitution der Verbindungen.}

Die Konstitutionsbestimmung von Siliciden haben bereits $\mathrm{M}_{\text {ANснот }}$ und KIESER ${ }^{1}$ unternommen, wobei sie hauptsächlich die Wasserstoffmenge, welche von dem Silicid, Doppelsilicide von Crom und Aluminium, beim Zersetzen mit Flufssäure resp. Flufssäure und Natronlauge entwickelt wurde, zugrunde legten. Für die vorliegenden Silicide konnte nun anfserdem das Verhalten derselben gegen Salzsäure und Stickstoff zur Aufstellung einer Konstitutionsformel herangezogen werden. Unter Berücksichtigung des Gesamtverhaltens gelangt man für das Silicid I zu folgendem Formelbild:

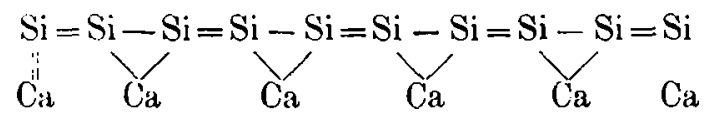

Der Zerfall im Stickstoffstrom geht dann in der Weise vor sich, dafs der an den beiden Enden sich befindliche Komplex von drei Siliciumatomen abgespalten wird, wobei sich vielleicht die folgenden Verbindungen bilden können:
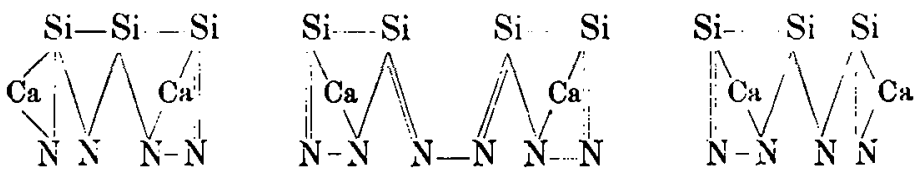

Der mittlere Körper würde dabei den mit Salzsäure nicht zersetzbaren Anteil und die beiden äufseren den zersetzbaren Anteil des Produktes bilden. Bei der Aufstellung dieser Konstitutionsformein mufste ror allen Dingen dem Umstand Rechnung getragen werden, dafs in dem einen Produkt das Calcium und der Stickstoff mit Salzsäure zchwer abspaltbar sind, während sie in dem anderen Produkt zum Teil leicht abgespalten werden können.

Durch Einwirkung von Salzsäure entsteht aus dem Silicid I ein gelbes Silikon von der Formel $\mathrm{Si}_{10} \mathrm{H}_{12} \mathrm{O}_{8}$ oder $\mathrm{Si}_{10} \mathrm{H}_{10} \mathrm{O}_{8}$. Die Bildung der Silikone wird in der Weise vor sich gehen, dals zunächst wie bei den Carbideu das Metall unter Bildung eines kompliziert zu-

1 Dissertation Würzburg 1906. 
sammengesetzten Siliciumwasserstoffs abgespalten wird. Wie schon der einfache Siliciumwasserstoff zeigt, sind diese Körper sehr unbeständig und oxydieren sich leicht. Für die Formel der Silikone kommt weiter noch in Betracht, daf's sie bei Abschlufs von Sauerstoff in der Hitze ziemlich beständig sind. Das bei gewöbnlicher Temperatur getrocknete Silikon verliert beim Erwärmen bis $120^{\circ}$ $7.4 \%$ Wasser, was einem Verluste von zwei Molekülen $\mathrm{H}_{2} \mathrm{O}$ entspricht. In den Silikonen wird also der Wasserstoff und Sauerstoff sich wahrscheinlich nicht völlig in Form von Hydroxylgruppen vorfinden, da dann eine leichtere Anhydrisierung eintreten könnte. Nach diesen Gesichtspunkten käme für das bei gewöhnlicher 'T'emperatur getrocknete Silikon I folgende Formel in Betracht:

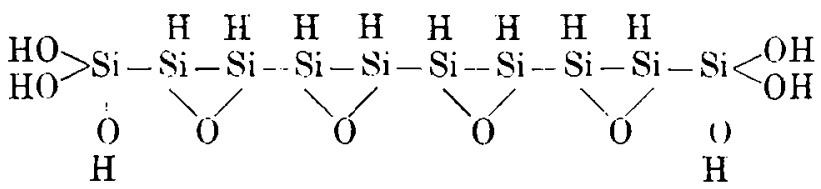

Bei $120^{\circ}$ werden zwei Moleküle Wasser abgespalten und es entsteht dann der folgende Körper:

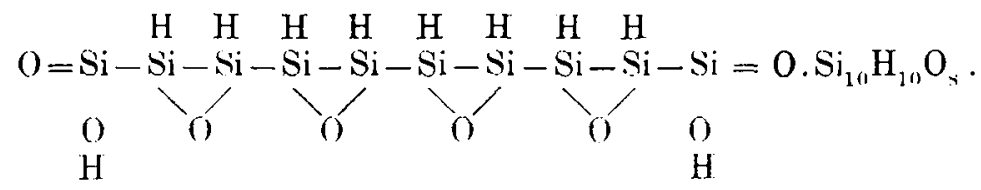

Die Analysenresultate des Silicids II waren nicht so konstant, wie bei Silicid I. Es ist nicht leicht, diesen Körper in reiner Form zu bekommen. Die Formeln, die sich aus den Analysen berechnen, liegen zwischen den Grenzen $\mathrm{Ca}_{14} \mathrm{Si}_{10}$ und $\mathrm{Ca}_{11} \mathrm{Si}_{10}$. Die schwankenden Resultate scheinen neben den mehr oder minder grol'sen Verunreinigungen an oxydierten Substanzen daher zu kommen, dafs sich das Silicid in dem überschüssigen Calcium löst und dieses beim Hrkalten mit auskrystallisiert. Je nachdem sich nun die Masse rascher oder langsamer abkühlt, werden die Krystalle mebr oder weniger freies Calcium eingeschlossen balten. Je langsamer die Masse sich abkühlt, desto reiner werden die Krystalle sein. Es werden also die Aualysen, die den höchsten Siliciumgehalt haben, am wahrscheinlichsten sein; diese Resultate nähern sich der Formel $\mathrm{Ca}_{11} \mathrm{Si}_{10}$. Diese Formel lälst sich auch am besten mit der vorhergehenden Formel für das Silicid $I$ in Einklang bringell. Bei dem Silicid II sind von den 40 Siliciumvalenzen 22 durch Calcium abgesättigt; es 
verbleiben also noch 18 , die unter sich abgesättigt sind. In dem Molekül sind demnach 9 einfache Bindungen vorhanden und es ergibt sich dann die Formel:

$$
\mathrm{Ca}=\underbrace{\mathrm{Si}}_{\mathrm{C}}-\mathrm{Si}-\mathrm{Si}-\mathrm{Si}-\mathrm{Si}-\mathrm{Si}-\mathrm{Si}-\mathrm{Si}-\mathrm{Si}-\mathrm{Si}=\mathrm{Ca}
$$

In dieser Formel sind alle Doppelbindungen des Silicid I durch Calciumatome abgesättigt.

Mit Salzsäure entsteht aus diesem Silicid neben Siliciumwasserstoff ein weifses Silikon von der Formel $\mathrm{Si}_{8} \mathrm{H}_{7} \mathrm{O}_{13}$ oder $\mathrm{Si}_{8} \mathrm{H}_{8} \mathrm{O}_{13}$. Diese Abspaltung von Siliciumwasserstoff zeigt, dafs durch den Eintritt des Calciums in das Molekül die Bildung zwischen den einzelnen Siliciumatomen lockerer geworden ist und dafs jetzt durch Einwirkung von Salzsäure eine leichte Sprengung der Kette stattfinden kann. Auch aus dem Sauerstoffgehalt der Silikone geht herror, dals die Siliciumkette bier durch Sauerstoffi wahrscheinlich unterbrochen ist. Wenn man absieht von einer weitgehenden Spaltung der Siliciumkette, kommt man für das Silikon II zur Formel:



Für die stickstoffhaltigen Körper aus dem Silicid II lälst sich nach den erhaltenen Werten eine einfache Formel schwer ermitteln.

Mit Hilfe der beschriebenen Stickstoff-Silicium-Calciumverbindungen ist ein neuer Weg gezeigt, den Stickstoff der luaft in verwertbarer Form zu gewionen. An und für sich sind diese stickstoffhaltigen Produkte ein wertrolles Düngemittel, die unter Umständen den Kalkstickstoff übertreffen können. Denn einmal würde dieser Körper keinerlei Giftwirkungen ausüben können und dann ist die Stickstoffabgabe dieses Körpers eine sehr langsame; es würde das Produkt eine sparsame und lang anhaltende Stickstoffquelle in Ackerboden sein.

Weiterhin lassen sich diese Verbindungen durch Schmelzen mit Soda oder Soda und Kohle in Cyannatrium verwenden.

Larmstadt, Techn. Hochschule. Laboratorium für allgemeine Chemie.

Bei der Redaktion eingegangen am 8. Oktober 1909. 